\title{
Strange form factors of the nucleon in the chiral quark-soliton model
}

\author{
A. Silva ${ }^{1}$ a, D. Urbano ${ }^{1,2}$, H.-C. Kim ${ }^{3}$, and K. Goeke ${ }^{4}$ \\ 1 Centro de Física Computacional, Departamento de Física da Universidade de Coimbra, P-3004-516 Coimbra, Portugal \\ 2 Faculdade de Engenharia da Universidade do Porto, R. Dr. Roberto Frias s/n, P-4200-465 Porto, Portugal \\ 3 Department of Physics, Pusan National University, 609-735 Pusan, Republic of Korea \\ 4 Institut für Theoretische Physik II, Ruhr-Universität Bochum, D-44780 Bochum, Germany
}

Received: 15 October 2004 / Published Online: 8 February 2005

(C) Società Italiana di Fisica / Springer-Verlag 2005

\begin{abstract}
The results for the strange electromagnetic and axial form factors in the chiral quark-soliton model are reviewed. The roles of a new quantization method and of the meson asymptotic behaviour are discussed. Predictions for the A4 and G0 experiments are presented.
\end{abstract}

PACS. 12.40.-y Other models for strong interactions - 14.20.Dh Protons and neutrons - 13.40.Gp Electromagnetic form factors

\section{Introduction}

The question of the strange content of the nucleon is a very important one in the understanding of hadron structure, which eventually is to be settled by experiment. Indeed, on one hand there is no obvious theoretical reason why strange $s \bar{s}$ pairs should not contribute to the nucleon properties. On the other hand the theoretical mechanism and the actual contribution of strange quarks are not yet understood as well.

Early results about the strange quark bilinears in the nucleon included the analysis of the sigma term [1] and polarized DIS. These first results indicated strange quark nonvanishing contributions to the nucleon's mass and spin, respectively. The strange vector currents associated with electromagnetic form factors were discovered to be accessible by means of weak neutral current experiments [2,3, 4]. For reviews see [5].

Due to its importance, the question of the strange form factors has been addressed before in the context of the chiral quark-soliton model (CQSM) 7,8, This work reviews the CQSM results 910] for the strange electromagnetic form factors and presents also the strange axial form factor. These form factors are obtained using a new quantization method [11] and studied phenomenologically in terms of the effects of the asymptotic behavior of the meson fields.

\footnotetext{
a Financial support from the organization of PAVI04 and from the Center for Computational Physics (CFC) for the participation in PAVI04 is kindly acknowledged. The work of A.S. has been partially supported by the grant PRAXIS XXI $\mathrm{BD} / 15681 / 98$.
}

\section{The chiral quark-soliton model (CQSM)}

The chiral quark-soliton model (CQSM) has been applied successfully to the description of many baryon observables, both in flavours $\mathrm{SU}(2)$ and $\mathrm{SU}(3)$. See [12]13] for reviews. The Lagrangian of the CQSM reads,

$$
\mathcal{L}=\bar{\psi}(x)\left(i \not \partial-m-M U^{\gamma_{5}}\right) \psi(x)
$$

where $m=\operatorname{diag}\left(m_{u}, m_{d}, m_{s}\right)$ is the current quark mass matrix and $M$ the constituent quark mass. $M$ is the only free parameter in the model. The main property of the model in relation with hadron physics is chiral symmetry and its spontaneous breaking. The auxiliary chiral fields $U^{\gamma_{5}}=e^{i \gamma^{5} \pi \cdot \tau}$ in (1), may be interpreted as physical meson fields and, thus, the Lagrangian (11) corresponds to a theory of constituent quarks interacting through the exchange of mesons.

However, the field description embodied in (1) is not renormalizable. A regularization method is therefore necessary to completely define the model. This work uses the proper-time regularization, which introduces one cutoff parameter into the model. The remaining parameters of the model are the current quark masses. In this work isospin breaking effects have been neglected and therefore the current mass parameters are the equal current masses $(\bar{m})$ of the $u$ and $d$ quarks and the current mass $\left(m_{s}\right)$ of the strange quarks. In order to fit these parameters, one calculates the pion and kaon masses as well as the pion decay constant in terms of the parameters of the model. Equating the model results for these quantities with their experimental values allows to fix the parameters of the model: the current nonstrange quark masses, the strange quark mass and the cut-off come out around $8 \mathrm{MeV}, 164 \mathrm{MeV}$ and $700 \mathrm{MeV}$, respectively. 


\section{The model baryon state}

In broad terms, the construction of the model baryon states necessary to compute baryon matrix elements are obtained in a projection after variation procedure, with the variation referring to the mean field solution.

Taking the large $N_{c}$ limit of the correlation function of two baryon currents and neglecting the strange quark mass lead to an effective action from which the mean field solution is obtained. At this level the effective action is proportional to the energy of the mean field, $S_{\text {eff }}[U] \sim$ $\mathcal{M}[U]$, which is a functional of the chiral fields $U: \mathcal{M}[U]=$ $N_{c} \epsilon_{\mathrm{V}}[U]+\epsilon_{\mathrm{S}}[U]$, with $\epsilon_{\mathrm{V}}$ the energy of the valence level, occupied by $N c$ quarks, and $\epsilon_{\mathrm{S}}$ the regularized energy of the Dirac sea. Both result from the solution of the Dirac one-particle problem $\epsilon_{n}[U]=\langle n|h(U)| n\rangle$ with

$$
h(U)=-i \gamma^{0} \gamma^{i} \partial_{i}+\gamma^{0} M U^{\gamma_{5}}+\bar{m} \gamma^{0} .
$$

In order to solve such problem one further restricts the mesons to the hedgehog shape, $\pi \cdot \tau=P(r) \hat{n} \cdot \tau$, with a profile function $P(r)$ vanishing at large distances. The minimization of the action corresponds to a profile function $P_{c}(r)$ which represents the mean field soliton. The formalism in flavour $\mathrm{SU}(3)$ is built upon the embedding of the $\mathrm{SU}(2)$ hedgehog into an $\mathrm{SU}(3)$ matrix [14]:

$$
U=e^{i P(r) \sum_{a=1}^{8} n^{a} \lambda^{a}} \rightarrow\left(\begin{array}{cc}
e^{i P(r) \sum_{i=1}^{3} n^{i} \tau^{i}} & 0 \\
0 & 1
\end{array}\right)
$$

\subsection{Quantization}

The quantization of the mean field is achieved in the context of collective coordinates. The collective coordinates are the orientations of the soliton in configuration as well as in flavour spaces (related by the hedgehog) and the position of the center of mass. These coordinates are most suited to describe the rotational zero modes, i.e. unconstrained large amplitude motion, which should be treated exactly. The model calculation neglects modes normal to the zero modes, which are subjected to restoring forces and hence suppressed.

The time dependent solutions are constructed on the basis of the restriction to the zero modes by the ansatd 1

$$
U(\boldsymbol{x}) \rightarrow A(t) U_{c}(\boldsymbol{x}) A^{\dagger}(t)
$$

with the collective coordinates contained in $A$.

In the laboratory system the Dirac operator becomes

$$
D(U)=A\left[D\left(U_{c}\right)+A^{\dagger} \dot{A}+\gamma^{0} A^{\dagger} \delta m A\right] A^{\dagger},
$$

which clearly shows the two expansion parameters used in this work: the angular velocity $A^{\dagger} \dot{A}(t)=i \Omega_{E}^{\alpha} \lambda^{\alpha} / 2$ and the strange mass related parameter $\delta m$, discussed below, in Sect. 3.2, in connection with the asymptotic meson behaviour effects.

\footnotetext{
1 Not showing translational zero modes explicitly.
}

The collective coordinate Hamiltonian is

$$
H=\mathcal{M}\left(U_{c}\right)+H_{\text {coll }}^{\mathrm{sc}}+H_{\text {coll }}^{\mathrm{sb}},
$$

i.e. it is composed by a flavour symmetry conserving (sc) and a symmetry breaking ( $\mathrm{sb}$ ) pieces. While the first is treated exactly, the second is treated perturbatively. The wave functions of the symmetry conserving part are the Wigner $\mathrm{SU}(3)$ matrices. For the octet

$$
|B, 8\rangle^{\mathrm{sc}} \rightarrow \Psi_{\nu \nu^{\prime}}^{(8) \mathrm{sc}}(A)=\sqrt{8}(-)^{Y^{\prime} / 2+J_{3}} D_{\nu \nu^{\prime}}^{(8) *}(A) .
$$

with $\nu=\left(Y, T, T_{3}\right)$ and $\nu^{\prime}=\left(Y^{\prime}, J, J_{3}\right)$ standing for the quantum numbers of the baryon state $\left(Y^{\prime}=-1\right)$. The symmetry breaking part of the Hamiltonian leads to a representation mixing, in first order perturbation theory, from which the baryon wave function $|B, 8\rangle^{\mathrm{sc}}$ picks terms from higher order representations [14].

\subsection{Asymptotics of the meson fields and symmetry conserving quantization}

The question of the asymptotics of the meson fields originates in the fact that the embedding (3) forces all the meson fields to have a common asymptotic behavior. This is not satisfactory in the $\mathrm{SU}(3)$ case due to the large mass difference between pions and kaons. In order to have some information on how large these effects may be, one exploits in this work the fact that there is no prescription to fix the multiples of the unity mass matrices in (2) and in the symmetry breaking piece $\delta m$ of (5):

$$
\gamma^{0} D\left(U_{c}\right)+A^{\dagger} \delta m A=-i \gamma^{i} \partial_{i}+M U^{\gamma^{5}}+\bar{m}+A^{\dagger} \delta m A
$$

where $\delta m=M_{1}+M_{8} \lambda^{8}$, with $M_{1}=\left(m_{s}-\bar{m}\right) / 3$ and $M_{8}=\left(\bar{m}-m_{s}\right) / \sqrt{3}$. In this case one may obtain larger mass asymptotics of the meson fields, by, in (8), increasing $\bar{m}$ at the expense of a lower $M_{1}$. The pion asymptotics will be denoted in the following by $\mu \rightarrow \pi(\sim 140 \mathrm{MeV})$ and the kaon asymptotics by $\mu \rightarrow K(\sim 490) \mathrm{MeV})$.

The symmetry conserving quantization [11] used in this work avoids the problem which is encountered in this model by using the ansatz (4) to compute the relation between isospin $(T)$ and spin $(S)$ operators. While the result from (4) reads

$$
T_{\alpha}=-D_{\alpha \beta}^{(n)}(A) J_{\beta}-\sqrt{3} D_{\alpha 8}^{(n)}(A) \frac{I_{2}^{\prime}}{I_{2}},
$$

the correct relation implies $I_{2}^{\prime}=0$. The symmetry conserving quantization identifies the terms like $I_{2}^{\prime}$ in the expressions for observables on the basis of the quark model limit of the CQSM.

\section{Strange electromagnetic form factors}

The knowledge of the form factors of the octet vector currents is enough to provide the form factors for each flavour:

$$
\begin{aligned}
V_{\mu}^{0} & =\bar{u} \gamma_{\mu} u+\bar{d} \gamma_{\mu} d+\bar{s} \gamma_{\mu} s, \\
V_{\mu}^{3} & =\bar{u} \gamma_{\mu} u-\bar{d} \gamma_{\mu} d, \\
\sqrt{3} V_{\mu}^{8} & =\bar{u} \gamma_{\mu} u+\bar{d} \gamma_{\mu} d-2 \bar{s} \gamma_{\mu} s .
\end{aligned}
$$


Table 1. Strange magnetic moment (in n.m.) and electric and magnetic radii (in $\mathrm{fm}^{2}$ ). The constituent quark mass is $420 \mathrm{MeV}$ and the strange quark mass $180 \mathrm{MeV} . \pi$ and $K$ stand for the two asymptotic descriptions of the mesons as discussed in Sect. 3.2

\begin{tabular}{lrr}
\hline$\mu$ & $\pi$ & $\mathrm{K}$ \\
\hline$\left\langle r^{2}\right\rangle_{E}^{s}$ & -0.220 & -0.095 \\
$\mu_{s}$ & 0.074 & 0.115 \\
$\left\langle r^{2}\right\rangle_{M}^{s}$ & 0.303 & 0.631 \\
\hline
\end{tabular}

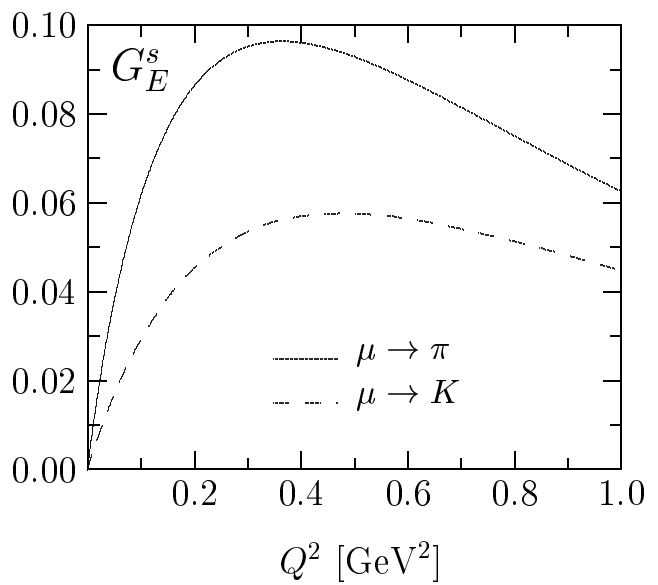

Fig. 1. Strange electric form factor of the nucleon. Conventions and model parameters as in Table 1

In this work the form factors for these currents were first obtained for the two asymptotic behaviours. The effects of the asymptotics was then studied at the level of baryon form factors by combining flavour form factors with pion asymptotics for nonstrange quarks with kaon asymptotics for strange form factors, i.e. by taking

$$
G_{E, M}^{B}\left(Q^{2}\right)=G_{E, M}^{u B(\pi)}\left(Q^{2}\right)+G_{E, M}^{d B(\pi)}\left(Q^{2}\right)+G_{E, M}^{s B(K)}\left(Q^{2}\right) .
$$

The most significant consequences of this ansatz for the baryon octet electromagnetic form factors is the improvement in the overall description of magnetic form factors and the neutron electric form factor. This supports the expectation that the strange form factors are better described in terms of kaon $(\mu \rightarrow K)$ rather than in pion $(\mu \rightarrow \pi)$ asymptotics. The results for $(\mu \rightarrow \pi)$ are nevertheless always presented.

The strange magnetic moment and the radii are presented in Table 1 and the strange electric and magnetic form factors in the CQSM are shown in Fig. [1 and Fig. 2] respectively. A particular aspect of these results is the positive value for the strange magnetic moment, which seems to be at variance with many theoretical calculations based in different approaches. The CQSM seems nevertheless to be able to accommodate higher values of this quantity, up to 0.41 n.m., as is found in the "model independent analysis" of [15]. This pinpoints the SU(3) structure of the rotational and mass corrections as the reason for such

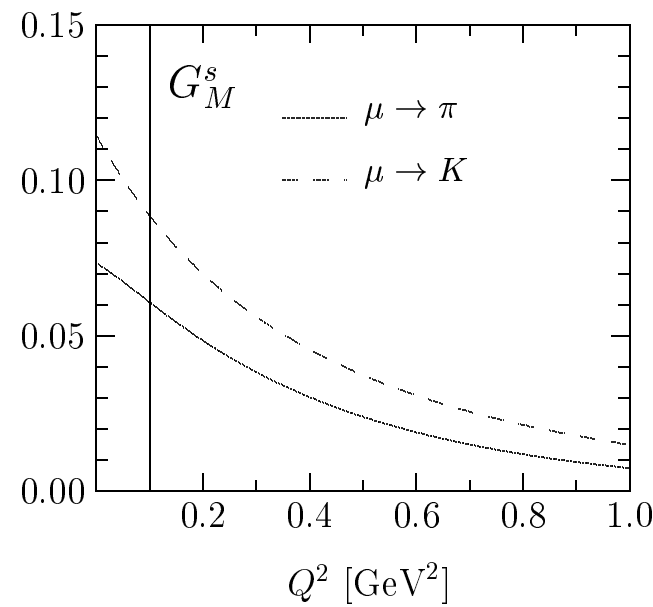

Fig. 2. Strange magnetic form factor in nuclear magnetons. Conventions and model parameters as in Table 1

positive value, similarly to what is observed in other models by maintaining just the $\mathrm{SU}(3)$ structure [16].

\subsection{The SAMPLE, HAPPEX, A4, and G0 experiments}

The experimental value for the magnetic form factor obtained by the SAMPLE collaboration [17] is

$$
G_{M}^{s}\left(Q^{2}=0.1\right)=+0.37 \pm 0.20 \pm 0.26 \pm 0.07 \text { (n.m.). }
$$

The CQSM results of this work underestimates this experimental result, still falling however within the error bar, shown in Fig. 2 at $Q^{2}=1$. The HAPPEX result [18

$$
\left(G_{E}^{s}+0.392 G_{M}^{s}\right)\left(Q^{2}=0.477\right)=0.014 \pm 0.030,
$$

is, on the contrary, overestimated in the CQSM, with the nearest result (kaon asymptotics) being 0.073.

Using the necessary form factors, one may study the combination $G_{E}^{s}\left(Q^{2}\right)+\beta\left(Q^{2}, \theta\right) G_{M}^{s}\left(Q^{2}\right)$, with $\beta\left(Q^{2}, \theta\right)=$ $\tau G_{M}^{p \gamma} / \epsilon G_{E}^{p \gamma}, \tau=Q^{2} /\left(4 M_{N}^{2}\right), \epsilon^{-1}=1+2(1+\tau) \tan ^{2}(\theta / 2)$, for the values of $\theta$ and $Q^{2}$ used in current or near future experiments. Figure 3 compares the model predictions with the recent forward data,

$$
\begin{aligned}
& \left(G_{E}^{s}+0.225 G_{M}^{s}\right)\left(Q^{2}=0.23\right)=0.039 \pm 0.034 \\
& \left(G_{E}^{s}+0.106 G_{M}^{s}\right)\left(Q^{2}=0.10\right)=0.074 \pm 0.036,
\end{aligned}
$$

with (16) from [19] and (17) from [20, by the A4 Collaboration and gives predictions for the backward angle. Figure 4 gives predictions for the G0 experiment.

\section{Strange axial form factor}

The axial currents of interest are expressed in term of flavour components in the same way as (12). The axial form factors for the octet axial currents have also been computed in the model with the same accuracy of the 


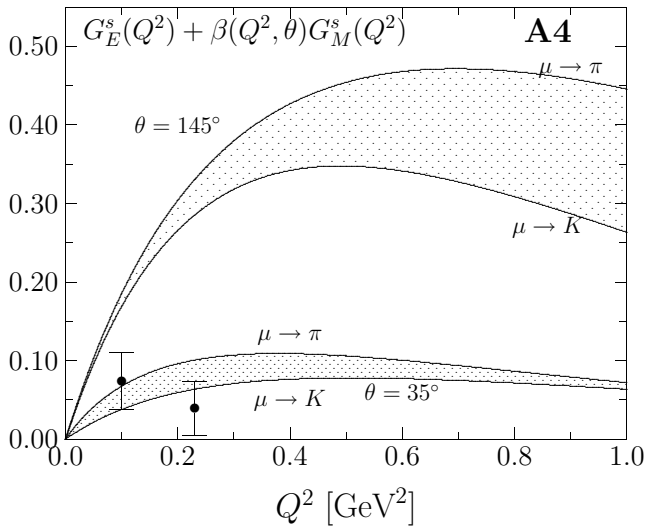

Fig. 3. Predictions of $G_{E}^{s}\left(Q^{2}\right)+\beta\left(Q^{2}, \theta\right) G_{M}^{s}\left(Q^{2}\right)$ for the A4 experiment. Conventions and model parameters as in Table 1

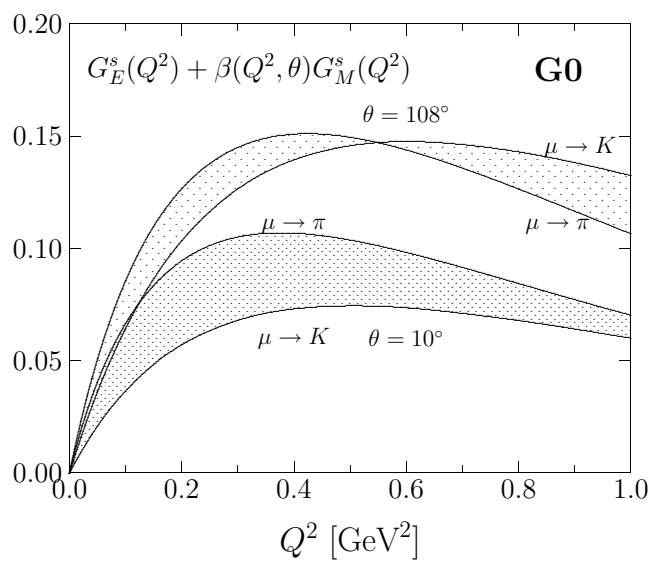

Fig. 4. Predictions for the G0 experiment. Conventions and model parameters as in Table 1 .

Table 2. $\Delta s$ and strange axial r.m.s. Conventions and model parameters as in Table 1

\begin{tabular}{lrr}
\hline & $\pi$ & $K$ \\
\hline$\Delta s$ & -0.086 & -0.075 \\
$\left\langle r_{s}^{2}\right\rangle^{1 / 2}$ & 0.554 & 0.172 \\
\hline
\end{tabular}

electromagnetic ones. The axial constants and axial dipole mass reproduce reasonably well the available experimental data. The results for $\Delta s=G_{A}^{s}\left(Q^{2}=0\right)$ and the strange axial r.m.s. are shown in Table 2 and the strange axial form factor is presented in Fig. 5. The CQSM results for $\Delta s$ seem to fall below a recent analysis of DIS data, which yields $\Delta s=-0.14 \pm 0.03[21]$.

\section{Conclusions}

The CQSM results for the strange form factors are not ruled out by the presently available experimental data. The results are stable from the point of view of the model parameters, with higher order corrections in $N_{c}$ and $\delta m$

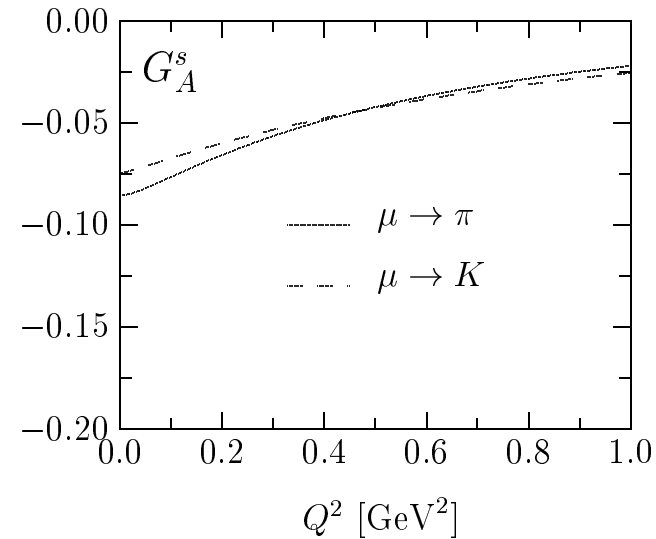

Fig. 5. Strange axial form factor. Conventions and model parameters as in Table[1]

expected below $15 \%$. The phenomenological observed effects related to the asymptotic behaviour of the meson fields requires nevertheless a more rigorous treatment.

\section{References}

1. J. Gasser, H. Leutwyler, M.E. Sainio: Phys. Lett. B 253 (1991) 252

2. D.B. Kaplan, A. Manohar: Nucl. Phys. B 310 (1988) 527

3. D.H. Beck: Phys. Rev. D 39 (1989) 3248

4. M.J. Musolf et al.: Phys. Rept. 239 (1994) 1

5. W.M. Alberico, S.M. Bilenky, C. Maieron: Phys. Rept. 358 (2002) 227 [arXiv:hep-ph/0102269]

6. D.H. Beck, R.D. McKeown: Ann. Rev. Nucl. Part. Sci. 51 (2001) 189 [arXiv:hep-ph/0102334]

7. H.C. Kim, T. Watabe, K. Goeke: Nucl. Phys. A 616 (1997) 606 [arXiv:hep-ph/9606440].

8. H. Weigel, A. Abada, R. Alkofer, H. Reinhardt: Phys. Lett. B 353 (1995) 20 [arXiv:hep-ph/9503241]

9. A. Silva, H.C. Kim, K. Goeke: Phys. Rev. D 65 (2002) 014016 [Erratum-ibid. D 66 (2002) 039902] [arXiv:hep$\mathrm{ph} / 0107185]$

10. A. Silva, H.C. Kim, K. Goeke: arXiv:hep-ph/0210189, to appear in EPJA

11. M. Praszalowicz, T. Watabe, K. Goeke: Nucl. Phys. A 647 (1999) 49 [arXiv:hep-ph/9806431]

12. C.V. Christov et al.: Prog. Part. Nucl. Phys. 37 (1996) 91 [arXiv:hep-ph/9604441]

13. D. Diakonov, V.Y. Petrov: arXiv:hep-ph/0009006

14. A. Blotz et al.: Nucl. Phys. A 555 (1993) 765

15. H.C. Kim, M. Praszalowicz, M.V. Polyakov, K. Goeke: Phys. Rev. D 58 (1998) 114027 [arXiv:hep-ph/9801295]

16. S.T. Hong, B.Y. Park, D.P. Min: Phys. Lett. B 414 (1997) 229 [arXiv:nucl-th/9706008]

17. D.T. Spayde et al. (SAMPLE Collaboration): Phys. Lett. B 583 (2004) 79 [arXiv:nucl-ex/0312016]

18. K.A. Aniol et al. (HAPPEX Collaboration): Phys. Rev. C 69 (2004) 065501 [arXiv:nucl-ex/0402004]

19. F.E. Maas et al. (A4 Collaboration): Phys. Rev. Lett. 93 (2004) 022002 [arXiv:nucl-ex/0401019]

20. F.E. Maas (for the A4 Collaboration): these proceedings

21. B.W. Filippone, X.D. Ji: Adv. Nucl. Phys. 26 (2001) 1 [arXiv:hep-ph/0101224] 